\title{
Differentiating the contributions between localized surface plasmon and surface plasmon polariton on a one-dimensional metal grating in coupling with a light emitter
}

\author{
Wen-Hung Chuang, Jyh-Yang Wang, C. C. Yang, ${ }^{a}$ and Yean-Woei Kiang ${ }^{\text {b) }}$ \\ Department of Electrical Engineering, Institute of Photonics and Optoelectronics, National Taiwan \\ University, 1, Roosevelt Road, Section 4, Taipei 10617, Taiwan
}

(Received 4 February 2008; accepted 15 March 2008; published online 3 April 2008)

\begin{abstract}
The authors demonstrate the differentiation between the contributions of localized surface plasmon (LSP) and surface plasmon polariton (SPP) couplings with an emitting dipole to emission enhancement in a metallic grating structure. Because of the relatively higher loss and in-plane radiation of the SPP modes, the LSP modes dominate the enhancement effect. However, because the LSP resonance energy is sensitive to the metal/dielectric interface geometry, it may be difficult to precisely implement a particular geometry and achieve the emission enhancement of a desired emission wavelength based on the LSP coupling. On the other hand, because the SPP feature can be controlled by the period of a grating structure, the implementation of the SPP coupling for emission enhancement in a practical device can be more feasible. (c) 2008 American Institute of Physics.

[DOI: $10.1063 / 1.2906363$ ]
\end{abstract}

The coupling of surface plasmons (SPs), including surface plasmon polariton (SPP) and localized surface plasmon (LSP), with light emitters/absorbers for enhancing emission/ absorption efficiency has been widely studied. ${ }^{\mathrm{I}-5}$ Among those studies, the coupling between a SP and an InGaN/GaN quantum well is quite attractive because it has the potential for increasing the efficiency of a light-emitting diode ${ }^{6-10} \mathrm{In}$ such a coupling process, dipole energy in a quantum well is transferred into a SP mode. If the SP mode can radiate effectively, light emission can be enhanced; otherwise, SP dissipation leads to emission quenching. In this regard, a metallic grating structure is useful for phase matching a SP mode and a radiation mode. ${ }^{11,12}$ However, in a metallic grating structure, besides SPPs of propagating nature can be excited, LSP modes can exist in individual grooves. The LSPs can also couple with the dipoles in the quantum well for either enhancing or quenching emission. Therefore, for enhancing the emission of a device, it is important to differentiate the coupling of a dipole with SPP from that with LSP in a metallic grating structure.

In this letter, we report the numerical study results of differentiating the SPP coupling with an emitting dipole from the LSP coupling. We are particularly interested in the contributions of SPP and LSP couplings to the emission enhancement factor and the enhancement spectral ranges. We use the boundary integral-equation method ${ }^{13}$ to numerically simulate the coupling between a dipole and all possible SP modes on a nearby $\mathrm{Ag}$ grating structure. We can observe the individual contributions of SPP and LSP to emission enhancement.

Figure 1 shows the spectra of the enhancement factor of bottom emission caused by the coupling of a dipole with a LSP in three cases of different groove shapes described by a super-Gaussian function. The three geometries of the $\mathrm{Ag} / \mathrm{GaN}$ interface are shown in the insert for the cases of A-C. The $x$-axis-oriented emitting dipole, denoted by an arrow and labeled by $J_{x}$, is located $10 \mathrm{~nm}$ right below the

\footnotetext{
${ }^{a}$ Electronic mail: ccy@cc.ee.ntu.edu.tw.

${ }^{b)}$ Electronic mail: ywkiang@ntu.edu.tw.
}

bottom of the groove with the coordinate at $(x, y)=(0$, $-20 \mathrm{~nm}$ ). Except the geometry of the semiconductor groove, the half-space $\mathrm{Ag}$ and the half-space $\mathrm{GaN}$ (with the refractive index set at 2.5) are divided by a plane interface at $y=0$. The groove and the dipole are infinitely extended along the $z$ axis to form the two-dimensional problem. The permittivity of $\mathrm{Ag}$ is assumed to follow the Drude model ${ }^{14}$ with the angular plasma frequency set at $\omega_{p}=1.19 \times 10^{16} \mathrm{rad} \mathrm{s}^{-1}$ and damping constant set at $\gamma=1.33 \times 10^{14} \mathrm{rad} \mathrm{s}^{-1}$, leading to the SPP resonance energy of $2.92 \mathrm{eV}$ (425 nm in wavelength) at the plane $\mathrm{Ag} / \mathrm{GaN}$ interface. With the half space of $\mathrm{Ag}$, the bottom emission (along the $-y$ direction) represents the total detectable power radiated from the dipole. The enhancement factor of bottom emission is defined as the total downward ( $-y$ direction) power flow across the surface of $y=-60 \mathrm{~nm}$ divided by the similar total power flow when the $\mathrm{Ag} / \mathrm{GaN}$ interface in the insert of Fig. 1 is replaced by a plane surface between air and $\mathrm{GaN}$ at $y=0$. This factor represents the increase of detectable power when the dipole is coupled with SPs induced on the $\mathrm{Ag} / \mathrm{GaN}$ interface. In Fig. 1, one can see the strong dependence of the enhancement factor spectrum

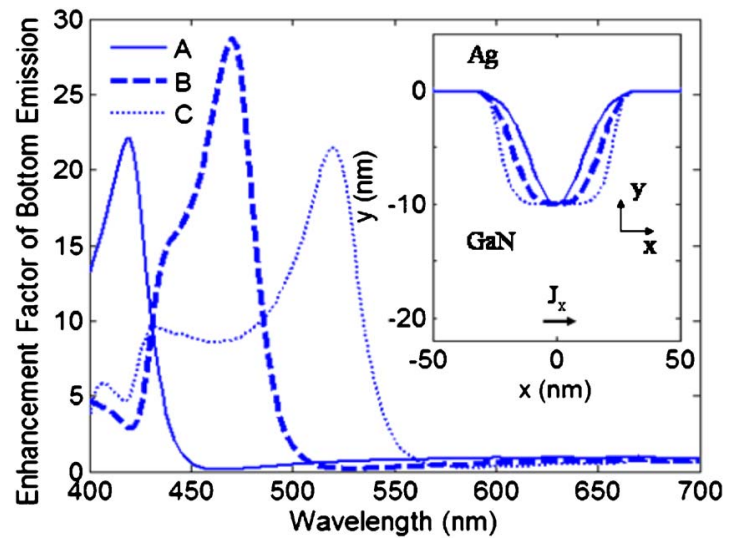

FIG. 1. (Color online) Spectra of the enhancement factor of bottom emission in three cases of different groove geometries. The $\mathrm{Ag} / \mathrm{GaN}$ interface geometries are shown in the insert for the cases of A-C. The $x$-axis-oriented dipole, denoted by an arrow and labeled by $J_{x}$, is located at $(x, y)=(0,-20 \mathrm{~nm})$. 

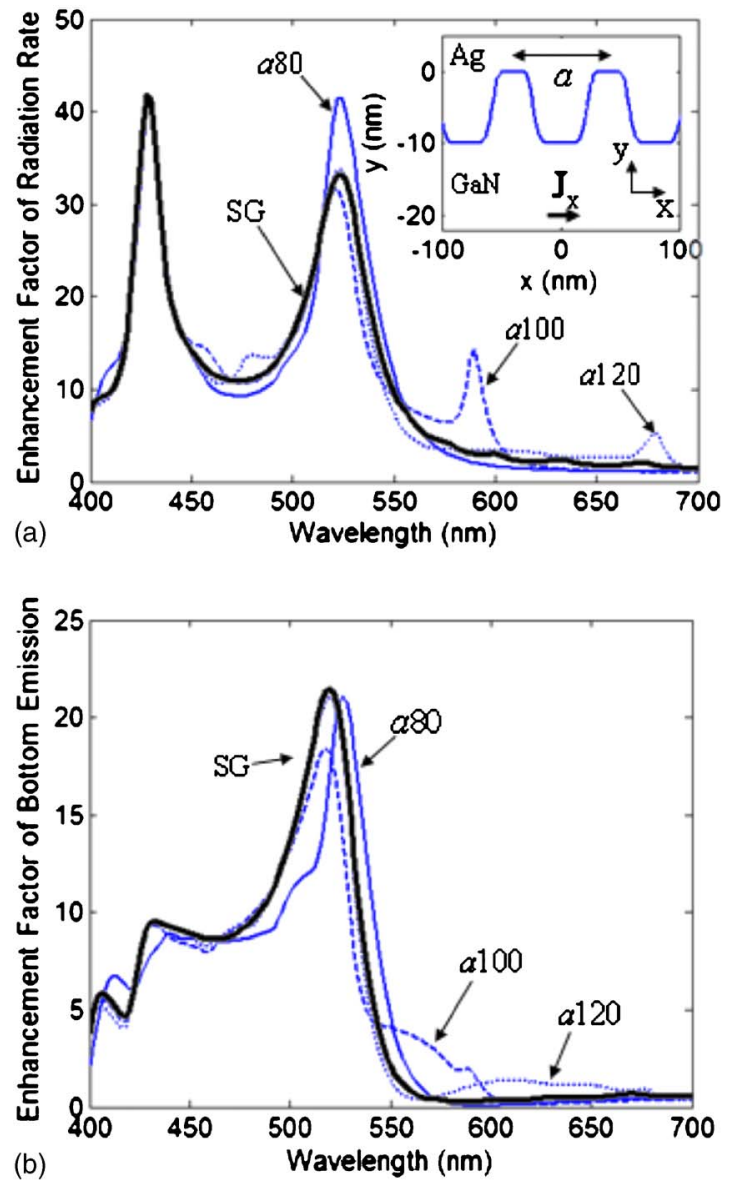

FIG. 2. (Color online) Spectra of the enhancement factor of radiation rate (a) and bottom emission (b) of four cases, including the single groove (denoted by SG) of case C in Fig. 1, the periodical arrangement of the groove to form the grating structures of periods $a=80 \mathrm{~nm}(a 80), 100 \mathrm{~nm}(a 100)$, and $120 \mathrm{~nm}(a 120)$. The grating structure is depicted in the insert of Fig. 2(a). on the groove geometry. The resonance energies of the LSPs in the groove shapes of A-C range from 420 to 470 up to $525 \mathrm{~nm}$ in wavelength, as depicted by the major peaks of the three curves in Fig. 1. The minor peaks of cases B and C around $430 \mathrm{~nm}$ are attributed to the resonant SPP mode on the flat $\mathrm{Ag} / \mathrm{GaN}$ interface. For case $\mathrm{A}$, this SPP feature shows itself as a shoulder of the dominating LSP resonance feature.

Figure 2(a) shows the spectra of the enhancement factor of radiation rate in four cases, including the single groove (denoted by SG) of case $\mathrm{C}$ in Fig. 1 and the periodical arrangement of the groove to form the grating structures of periods $a=80 \mathrm{~nm} \mathrm{(a80),} 100 \mathrm{~nm} \mathrm{(a100),} \mathrm{and} 120 \mathrm{~nm}$ (a120). The grating structure is depicted in the insert of Fig. 2(a), in which a dipole is located under one of the grooves, still $10 \mathrm{~nm}$ below the groove bottom. The enhancement factor of radiation rate is defined as the total outgoing power from a square of $2.5 \mathrm{~nm}$ in dimension centered at the dipole position divided by the similar power when the grating structure is replaced by a plane air/GaN interface at $y=0$. In Fig. 2(a), the curve of SG is similar to that of case C in Fig. 1, with the peaks around 525 and $430 \mathrm{~nm}$ corresponding to the induced LSP in the groove and the plane-interface-induced SPP resonance, respectively. Among the three grating structures, the aforementioned two peaks of the SG structure at 430 and $525 \mathrm{~nm}$ can also be observed, as expected. Besides, additional peaks due to the coupling of SPP with the dipole can be seen with the peaks around $590 \mathrm{~nm}$ for the case of $a=100 \mathrm{~nm}$ and around $680 \mathrm{~nm}$ for the case of $a=120 \mathrm{~nm}$. The SPP-coupling peak for the case of $a=80 \mathrm{~nm}$ merges into the LSP feature around $525 \mathrm{~nm}$ such that this peak shows a higher intensity. These peaks correspond to the dispersion curve interceptions with the light line (see Fig. 4 below).

Figure 2(b) shows the spectra of the enhancement factor of bottom emission for the same cases in Fig. 2(a). The difference between the bottom emission in Fig. 2(b) and the (total) radiation rate in Fig. 2(a) is mainly caused by the metal dissipation loss and in-plane $(y=0)$ light
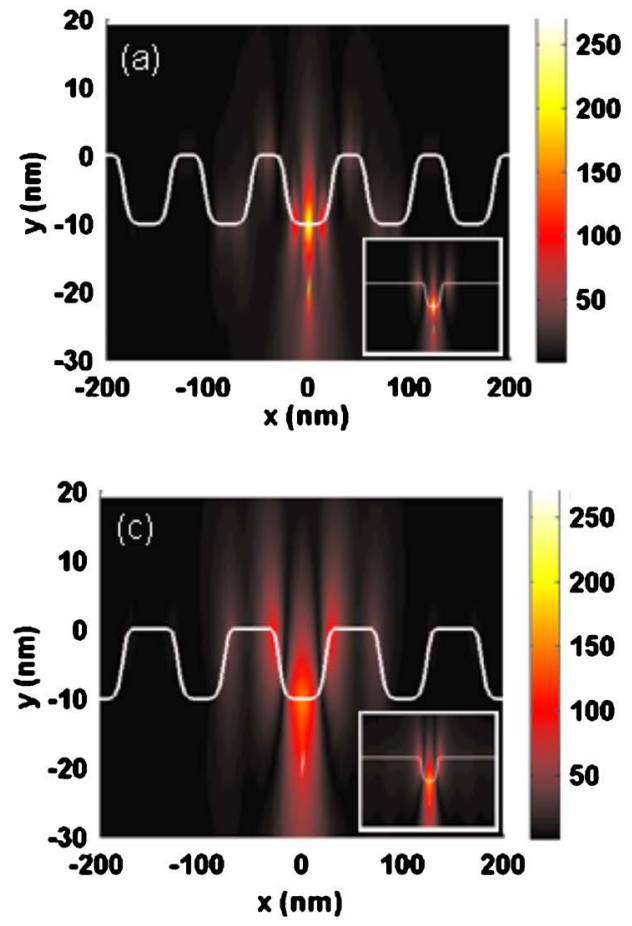
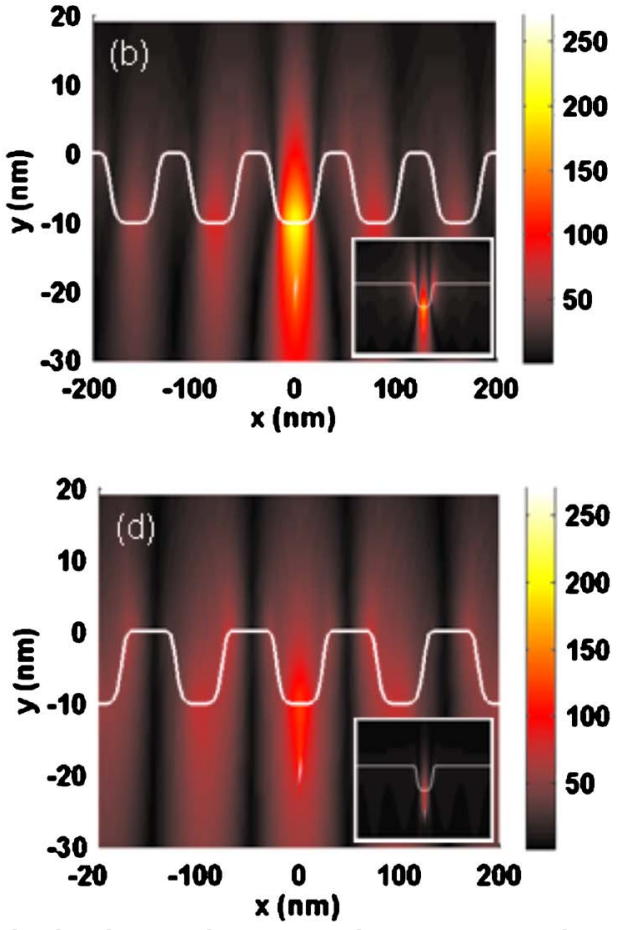

FIG. 3. (Color online) Distribution of the absolute value of $H_{z}$ field for the SP modes in the grating structure of $a=80 \mathrm{~nm}$ at $430 \mathrm{~nm}$ (a) and $525 \mathrm{~nm}$ (b), and in that of $a=100 \mathrm{~nm}$ at $525 \mathrm{~nm}(\mathrm{c})$ and $590 \mathrm{~nm}$ (d). The inserts of Figs. 3(a)-3(d) show the field distributions of the SG structure at the same wavelengths. 

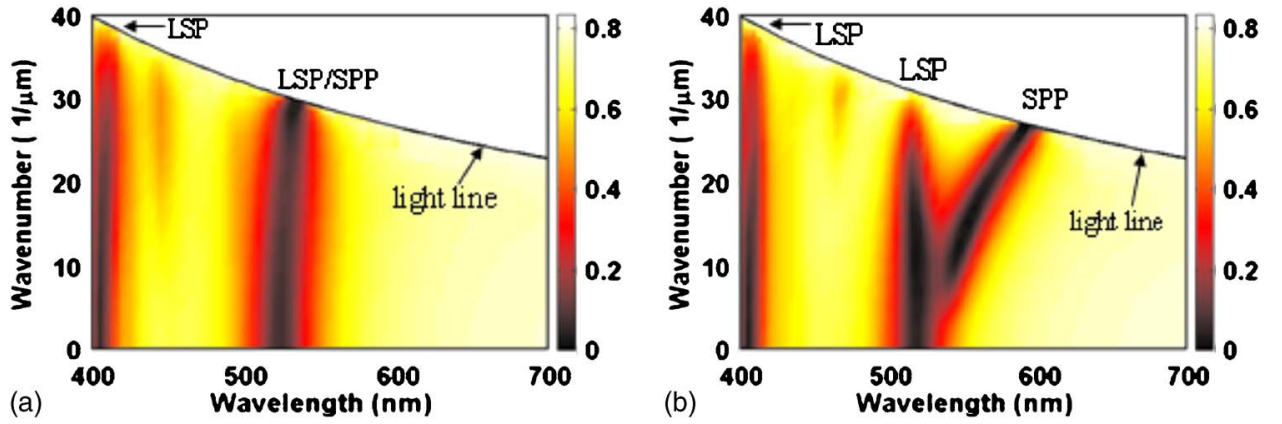

FIG. 4. (Color online) Dispersion curves of the grating structures with $a=80 \mathrm{~nm} \mathrm{(a)} \mathrm{and} 100 \mathrm{~nm}(\mathrm{~b})$. propagation. ${ }^{12}$ In Fig. 2(b), because of the high dissipation loss at the SPP resonance, the peaks of all cases around $430 \mathrm{~nm}$ are suppressed. With this suppression, the minor peaks around $405 \mathrm{~nm}$ become visible. This minor peak exists in all cases implying that it is generated by an individual groove. It can be attributed to a higher-order LSP mode in the groove. In Fig. 2(b), one can also observe the suppressions of the SPP peaks in the grating structures when compared with Fig. 2(a). The metal dissipation and in-plane light propagation result in a broad spectral hump for bottom emission enhancement [instead of the sharp peaks in Fig. 2(a)] in the SPP coupling with the dipole when the grating periods are 100 and $120 \mathrm{~nm}^{12}$ Such a suppression in the case of $a 80$ leads to the decreased peak intensity around $525 \mathrm{~nm}$, when compared with its counterpart in Fig. 2(a). Because the SPP feature, except that around $430 \mathrm{~nm}$, shifts with the grating period, we can differentiate the contribution of SPP couplinginduced emission enhancement from that of LSP. From the results in Figs. 2(a) and 2(b), one can realize that the contribution of LSP coupling can be more significant than that of SPP coupling if the dipole emission wavelength matches the LSP resonance.

To further understand the coupling between the dipole and the SP modes, we plot the distribution of the absolute value of $H_{z}$ field in Figs. 3(a)-3(d) for the SP modes in the grating structure of $a=80 \mathrm{~nm}$ at $430 \mathrm{~nm}$ (a) and $525 \mathrm{~nm}$ (b), and in that of $a=100 \mathrm{~nm}$ at $525 \mathrm{~nm}$ (c) and $590 \mathrm{~nm}$ (d). The inserts of Figs. 3(a)-3(d) show the field distributions of the SG structure at the same wavelengths. The SP field in Fig. 3(a) is localized, even though it corresponds to the resonant SPP on the plane $\mathrm{Ag} / \mathrm{GaN}$ interface, because the dissipation loss of SPP at the resonance energy is high such that its propagation distance is limited. The insert (also the resonant SPP mode) means to show the slight change of field distribution, particularly outside the central groove, when the periodical structure is formed. The field distribution in Fig. 3(b) consists of the SPP and LSP components in this grating structure. The insert in this figure shows the field of only the LSP in the SG structure. In Fig. 3(b), an extended distribution of SPP superimposed with the bright local field of LSP can be seen. From the total field distribution, one can speculate that the two SP modes are mutually coherent and constructively interfered when they couple simultaneously with the same dipole. In Fig. 3(c), one can see the field distribution of the LSP mode in the grating structure, which is different from that of the resonant SPP mode of limited propagation distance [see Fig. 3(a)] and is slightly more extended than the LSP mode in the SG structure. Then, in Fig. 3(d), we can observe a typical SPP modal field distribution which extends over several periods along the $y$ direction. Here, the insert shows that no LSP or SPP modes are excited.
Figures 4(a) and 4(b) show the dispersion curves of the grating structures with $a=80$ and $100 \mathrm{~nm}$, respectively, based on the reflection calculation of incident plane wave using the plane-wave-assisted boundary integral-equation method. ${ }^{13}$ The dark stripes indicate the dispersion curves of various SP modes. It is noted that the dispersion curve of the resonant SPP mode around $430 \mathrm{~nm}$, which is hardly excited due to the higher-order diffraction of the grating structure, cannot be clearly seen in Figs. 4(a) and 4(b). In Fig. 4(a), besides the mixed LSP/SPP modes around $525 \mathrm{~nm}$, the aforementioned higher-order LSP mode round $405 \mathrm{~nm}$ can be clearly observed. In Fig. 4(b), the SPP (around $590 \mathrm{~nm}$ ) and LSP (around $525 \mathrm{~nm}$ ) modes are separated that is consistent with the results in Fig. 2(a).

In summary, we have demonstrated the different contributions of LSP and SPP couplings with a dipole to emission enhancement. Because of the relatively higher loss and inplane radiation of the SPP modes, the LSP modes could dominate the enhancement effect. However, because the LSP resonance energy is sensitive to the groove geometry, it might be difficult to precisely implement a designed groove geometry and control the enhancement factor for a given emission wavelength.

This research was supported by National Science Council, The Republic of China, under Grant Nos. NSC 96-2120M-002-008 and NSC 96-2221-E-002-188, and by US Air Force Scientific Research Office under Contract No. AOARD-07-4010.

${ }^{1}$ A. Neogi, C. W. Lee, H. O. Everitt, T. Kuroda, A. Tacheuchi, and E. Yablonovitch, Phys. Rev. B 66, 153305 (2002).

${ }^{2}$ K. T. Shimizu, W. K. Woo, B. R. Fisher, H. J. Eisler, and M. G. Bawendi, Phys. Rev. Lett. 89, 117401 (2002).

${ }^{3}$ Y. Ito, K. Matsuda, and Y. Kanemitsu, Phys. Rev. B 75, 033309 (2007).

${ }^{4}$ K. Okamoto, S. Vyawahare, and A. Scherer, J. Opt. Soc. Am. B 23, 1674 (2006).

${ }^{5}$ M. A. Noginov, G. Zhu, M. Bahoura, C. E. Small, C. Davison, J. Adegoke, V. P. Drachev, P. Nyga, and V. M. Shalaev, Phys. Rev. B 74, 184203 (2006).

${ }^{6}$ K. Okamoto, I. Niki, A. Shvartser, Y. Narukawa, T. Mukai, and A. Scherer, Nat. Mater. 3, 601 (2004).

${ }^{7}$ D. M. Yeh, C. F. Huang, C. Y. Chen, Y. C. Lu, and C. C. Yang, Appl. Phys. Lett. 91, 171103 (2007).

${ }^{8}$ D. M. Yeh, C. F. Huang, Y. C. Lu, C. Y. Chen, T. Y. Tang, J. J. Huang, K. C. Shen, Y. J. Yang, and C. C. Yang, Appl. Phys. Lett. 91, 063121 (2007).

${ }^{9}$ Y. C. Lu, C. Y. Chen, D. M. Yeh, C. F. Huang, T. Y. Tang, J. J. Huang, and C. C. Yang, Appl. Phys. Lett. 90, 193103 (2007).

${ }^{10}$ C. Y. Chen, Y. C. Lu, D. M. Yeh, and C. C. Yang, Appl. Phys. Lett. 90, 183114 (2007).

${ }^{11}$ K. C. Shen, C. Y. Chen, C. F. Huang, J. Y. Wang, Y. C. Lu, Y. W. Kiang, C. C. Yang, and Y. J. Yang, Appl. Phys. Lett. 92, 013108 (2008).

${ }^{12}$ J. Y. Wang, Y. W. Kiang, and C. C. Yang, Appl. Phys. Lett. 91, 233104 (2007).

${ }^{13}$ J. Y. Wang, C. C. Yang, and Y. W. Kiang, Opt. Express 15, 9048 (2007).

${ }^{14}$ F. Wooten, Optical Properties of Solids (Academic, New York, 1972). 